\title{
Nanoparticles and Targeted Cancer Therapy
}

\author{
Yadav Ajay Kumar ${ }^{1,2 *}$, Gnawali Suman² and Yuan Gangbiao ${ }^{1}$ \\ ${ }^{1}$ Department of Nuclear Medicine, The Second Affiliated Hospital of Chongqing \\ Medical University, China \\ ${ }^{2}$ Department of Radio-Diagnosis, Imaging and Nuclear Medicine, BP Koirala \\ Memorial Cancer Hospital, Bharatpur, Chitwan, Nepal \\ *Corresponding Author: Yadav Ajay Kumar, Department of Nuclear Medicine, \\ The Second Affiliated Hospital of Chongqing Medical University, China. \\ DOI: $10.31080 /$ ASCB.2021.04.0344
}

Nanoscale devices are 100 to 10 thousand-fold smaller than human cells. They're similar in size to massive biological molecules ("biomolecules") like enzymes and receptors. As AN example, hemoglobin, the molecule that carries atomic number 8 in red blood cells, is close to five nanometers in diameter. Nanoscale devices smaller than fifty nanometers will simply enter most cells, whereas those smaller than twenty nanometers will move out of blood vessels as they flow into through the body. Attributable to their tiny size, nanoparticles will promptly perform with biomolecules on each the surface and within cells. By gaining access to numerous areas of the body, they need the potential to discover malady and deliver treatment in ways in which undreamt of previously.

These nerve cells are then given with the nano-vaccines (as shown during this image), that are porous chemical element particle discs loaded with immune stimulating molecules and growth antigens. These currently activated cells are then injected back to the host to stimulate an anti-tumor response.

Cancer therapies are presently restricted to surgery, radiation, and drugs therapy (Chemotherapy). All 3 ways risk harm to traditional tissues or incomplete demolition of the cancer. Technology offers the means that to focus on chemotherapies directly and by selection to cancerous cells and neoplasms, guide in surgical operation of tumors, and enhance the therapeutic effectuality of radiation-based and alternative current treatment modalities. All of this could add up to a bated risk to the patient and a multiplied likelihood of survival.

Research on technology cancer medical care extends on the far side drug delivery into the creation of latest medical special-
Received: August 27, 2021

Published: September 18, 2021

(C) All rights are reserved by Yadav Ajay

Kumar., et al. ty on the market solely through use of nanomaterial properties. Nanoparticles are too small as compare to human cells and are huge enough to encapsulate several tiny molecule compounds, which might be of several varieties. At constant time, the comparatively massive extent of nanoparticle are often functionalized with ligands, as well as little molecules, deoxyribonucleic acid or polymer strands, peptides, aptamers or antibodies. These ligands are often used for therapeutic impact or to direct nanoparticle fate in vivo. These properties alter mixture drug delivery, multi-modality therapy and joint therapeutic and diagnostic, referred to as "theranostic", achievement. The physical properties of nanoparticles, like energy absorption and re-radiation, may be accustomed disrupt pathological tissue, as in optical maser ablation and physiological state applications.

Integrated development of innovative nanoparticle packages and active pharmaceutical ingredients also will change exploration of a wider repertoire of active ingredients, now not confined to those with acceptable pharmokinetic or biocompatibility behavior. Additionally, immunogenic merchandise and surface coatings are being investigated as each adjuvant to nanoparticle-mediated and ancient radio- and therapy still as complete therapies. Innovative ways embrace the look of nanoparticles as artificial matter presenting cells and in vivo depots of immunostimulatory factors that exploit nanostructured design for sustained anti-tumor activity.

\section{Delivering therapy}

The traditional use of technology in cancer medical specialty has been to enhance the pharmacological medicine and scale back the general toxicities of chemotherapies through the selective tar- 


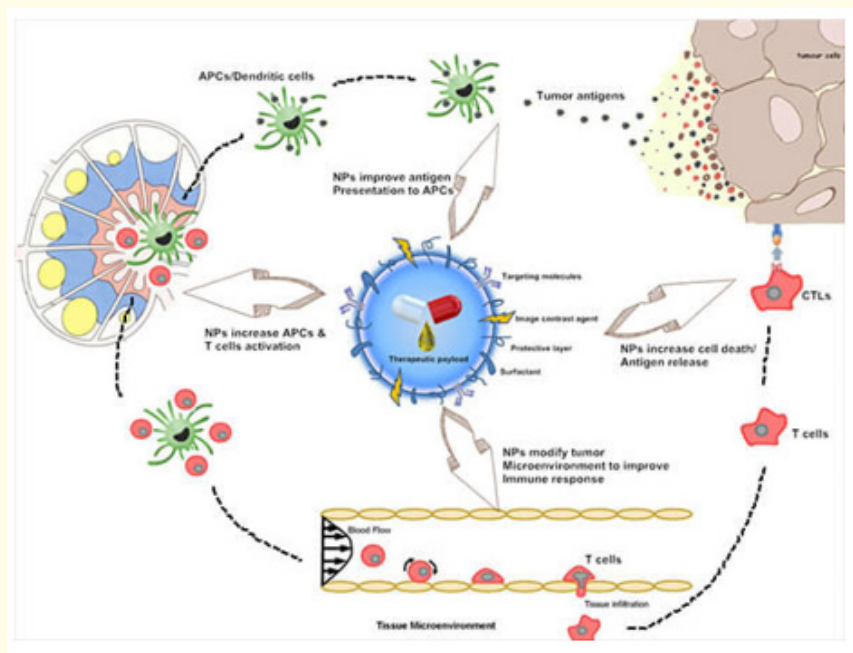

Figure 1: Depiction of the complex pathway involved in cancer immunotherapy. Nanoparticle delivery vehicles can play a role at multiple points along this pathway.

geting and delivery of those anticancer medicines to tumor. The advantage of nanosized carriers is that they'll increase the delivered drugs overall therapeutic index through nanoformulations in with chemotherapeutics are either encapsulated or conjugated to the surfaces of nanoparticles. This capability is essentially thanks to their tiny size and surface properties. Size could be a major consider the delivery of nanotechnology-based medical specialty to growth tissues. Selective delivery of nanotherapeutic platforms depends totally on the passive targeting of tumors through the improved permeableness and retention (EPR) impact. This development depends on defects specific to growth microenvironment like defects in humor drain, at the side of multiplied growth vasculature permeableness, to permit nanoparticles $(<200 \mathrm{~nm})$ to accumulate within the growth microenvironment. What is more, the temporal arrangement of drug unharness are often controlled by triggered events, like ultrasound, $\mathrm{pH}$, heat, or by material composition.

\section{Nano-enabled therapy}

Immunotherapy could be a promising new front in cancer treatment encompassing variety of approaches, as well as stop inhibition and cellular therapies. Though results for a few patients are spectacular, solely a minority of patients being treated for simply a set of cancers expertise sturdy responses to those therapies. Increasing the advantages of therapy needs a bigger understanding of tumor-host system interactions. New technologies for molecular and useful analysis of single cells are being employed to interrogate growth and immune cells and elucidate molecular indicators and useful immune responses to medical care. To the current finish, nano-enabled devices and materials are being leveraged to type, image, and characterize T cells within the Alliance's NanoSystems Biology Cancer Center.

This scanning microscope image shows nerve fibre cells, pseudo-colored in inexperienced, interacting with $\mathrm{T}$ cells, and pseudo-colored in pink. The nerve fibre cells interiorize the particles; method the antigens, and gift peptides to T cells to direct immune responses.

Nanotechnologies are being investigated to deliver therapy. This includes use of nanoparticles for delivery of immunostimulatory or immunomodulatory molecules together with chemo- or irradiation or as adjuvants to alternative immunotherapies. Standalone nanoparticle vaccines are being designed to lift ample lymph cell response to eradicate tumors, through co-delivery of matter and adjuvant, the inclusion of multiple antigens to stimulate multiple nerve fibre cell targets, and continuous unharness of antigens for prolonged immune stimulation.

Additional uses of technology for therapy embrace immune depots placed in or close to tumors for in place vaccination and artificial matter presenting cells. These and alternative approaches can advance and be refined as our understanding of cancer therapy deepens. Depiction of the complicated pathway concerned in cancer therapy. Nanoparticle delivery vehicles will play a job at multiple points on this pathway.

\section{Delivering or augmenting irradiation}

Roughly half all cancer patients receive some style of radiotherapy over the course of their treatment. Radiotherapy uses high-energy radiation to shrink tumors and kill cancer cells. Radiotherapy kills cancer cells by damaging their deoxyribonucleic acid causing cellular necrobiosis. Radiotherapy will either harm deoxyribonucleic acid directly or produce charged particles inside the cells which will successively harm the deoxyribonucleic acid. Most forms of radiation used for cancer treatment utilize X-rays, 
gamma rays, and charged particles. As such, they're inherently ototoxic to any or all cells, not simply cancer cells, and are given in doses that are as efficacious as doable whereas not being too harmful to the body or fatal. Attributable to this exchange between effectuality and safety relative to growth sort, location, and stage, usually the effectuality of treatment should stay at reduced levels so as to not be overtly ototoxic to close tissue or organs close to the growth mass.

Nanotechnology-specific analysis has been that specialize in irradiation as a treatment modality that would greatly take pleasure in nanoscale materials' properties and multiplied growth accumulation. The nanoscale platforms bank are either sweetening of the impact of the irradiation, augmentation of the medical care, and/ or novel outwardly applied radiation modalities. A lot of specifically, most of those technology platforms deem the interaction between radiation and nanoparticles thanks to inherent atomic level properties of the materials used. These embrace high- $Z$ number nanoparticles that enhance the Arthur Compton and physical phenomenon effects of standard radiotherapy. In essence, it is noted that increasing effectuality whereas maintaining this irradiation dose and its resultant toxicity to the encompassing tissue. Alternative platforms utilize radiation triggered drug-releasing nanoparticles that deliver drug regionally at growth web site or to sensitize the cancer cells to irradiation together with the drug.

Another form of medical care that depends upon external radiation is photodynamic medical care (PDT). It's a good anticancer| therapy for superficial tumor that depends on tumor localization of a photosensitizer followed by light-weight activation to get cytotoxic. Many nanomaterials platforms are being researched to the current finish. Usually product of a lanthanide- or hafnium-doped high-Z core, once injected these are often outwardly irradiated by radiation source permitting the nanoparticle core to emit the radiation local tumor site. Emission of photons from the particles after activate a nanoparticle-bound or native photosensitizer to get undershirt atomic number 8 (102) ROS for growth destruction. What is more, these nanoparticles are often used as each PDT that generates ROS and for increased radiotherapy via the high-Z core. Several of those platforms are at the start being studied in vivo by intratumoral injection for superficial growth sites; some are being tested for delivery via general injection to deep tissue tumors [1].

\section{End Note}

Nanotechnology is a powerful tool for combating cancer and is being put to use in other applications that may reduce pollution, energy consumption, greenhouse gas emissions, and help prevent diseases.

\section{Bibliography}

1. https://www.cancer.gov/nano/cancer-nanotechnology/ treatment

Volume 5 Issue 10 October 2021

(C) All rights are reserved by Yadav Ajay Kumar., et al. 